\title{
SUR LES FORMES KYSTIQUES DE GIARDIA VARANI
}

\author{
Par G. LAVIER
}

J'ai décrit, sous ce nom, en 1923, une espèce de Giardia rencontrée dans l'intestin d'un Varanus niloticus L. ; chez l'individu parasité, provenant de la région de Dakar, je n'avais pas observé de formes kystiques. Ayant eu, par la suite, l'occasion d'autopsier dix varans de la région d'Entebbé (Uganda), j'ai noté sept fois une infestation par ce flagellé qui est donc loin d'être rare.

Chez un seul individu, toutefois, j'ai rencontré des formes kystiques, très peu nombreuses, il est vrai ; mais il ne saurait néanmoins y avoir de doute quant à leur existence.

Les kystes de Giardia varani sont analogues, dans leur structure, à ceux qu'il est si facile d'observer chez les espèces du genre parasitant les mammifères. Cependant, chez le reptile, les kystes sont généralement plutôt sphéroïdes qu'ovalaires, et, en cela, se rapprochent de ceux de Giardia agilis des têtards, tels que les a dessinés Alexeieff (1914). Leur grand axe mesure de 8 à $11 \mu$; le petit axe de $7 \mu, 5$ à $9 \mu$.

La paroi est mince; de même que, dans les autres espèces du genre, on aperçoit, à l'intérieur, 2 ou 4 noyaux, un enchevêtrement compliqué de fibrilles, correspondant à l'appareil flagellaire de la forme végétative, et les filaments épais, recourbés et très sidérophiles qui formeront les fibrilles du pourtour de la « ventouse ».

L'intérêt de ces kystes ne repose donc pas dans leur structure, mais surtout dans leur existence. On connaît, en effet, actuellement, des représentants du genre Giardia chez de nombreux mammifères, chez plusieurs oiseaux; $G$. varani est encore la seule connue chez un reptile ; enfin, chez les amphibiens, existe l'espècetype du genre, G. agilis; il n'y a pas certitude de l'existence du genre chez des poissons. Or, si chez les Giardia de mammifères, on rencontre les kystes sans difficultẻ et le plus souvent même avec une abondance supérieure à celle des formes végétatives, on ne connaît pas encore de formes kystiques chez les Giardia d'oiseaux, et celles de G. agilis n'ont été signalées qu'une seule fois. Alexeieff les a rencontrées chez une très petite grenouille, peu après la métamorphose; il en a donné une description et des figures. Hegner (1922) toutefois, et Wenyon (1926, p. 711), en relatant la

Annales de Parasitologie, T. XII, $\mathrm{N}^{\circ} 1 .-1^{\mathrm{er}}$ janvier 1934, p. 56-57. 
découverte d'Alexeieff, font sentir un doute de leur part quant à l'attribution de ces formes à des kystes de Giardia.

Je ne crois cependant pas douteux qu'Alexeieff ait bien vu les formes kystiques de Giardia agilis; la comparaison des figures

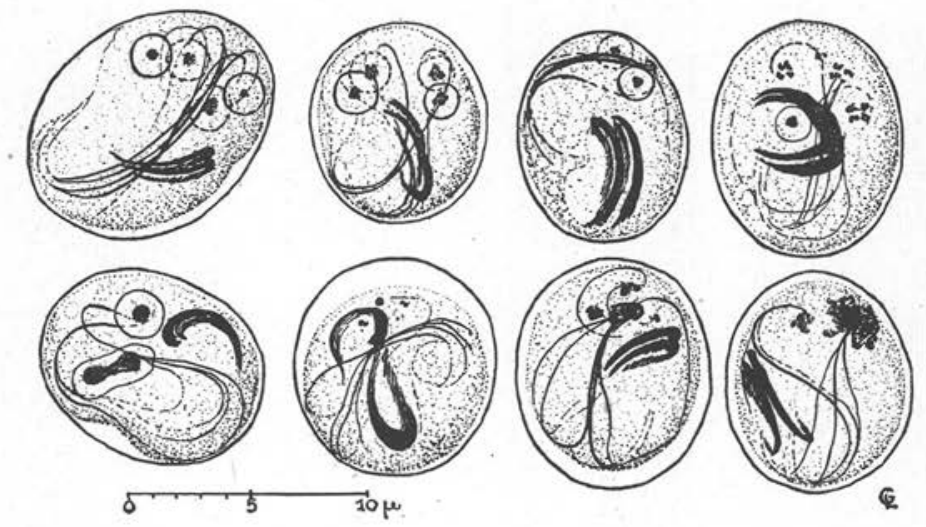

Fig. - Huit kystes de Giardia varani. Fix. Bouin ; col. Hématoxyline ferrique.

qu'il a données avec l'aspect des autres kystes du genre, et en particulier ceux de G. varani, me semble concluante à cet égard. L'extrême rareté de ces formes n'empêche pas la réalité de leur existence; elle prouve seulement que, chez l'amphibien comme chez le reptile, le déterminisme de leur apparition est entièrement différent de celui que l'on observe chez les mammifères.

\section{BiBLIOGRAPHIE}

AlexeiefF (A.). - Notes protistologiques. VIII. Sur la révision des genres de l'ordre Polymastigina Blochmann. Zool. Anz., XLIV, 1914, p. 211. (fig. 5).

Hegner (R. W.). - The Systematic Relationship of Giardia lamblia Stiles, 1915, from Man and Giardia agilis Kunstler, 1882, from the Tadpole. Amer. Journ. of Hyg., II, 1922, p. 435.

LAvier (G.). - Sur deux espèces nouvelles du genre Giardia : G. viscaciæ de la Viscache (Viscacia viscacia) et $G$. varani du Varan (Varanus niloticus). Ann. Parasitol., I, 1923, p. 147.

Wenyon (C. M.). - Protozoology, London, Baillière, Tindall and Cox, 1926, I, p. 711 .

Laboratoire de Zoologie et Parasitologie de la Faculté de médecine de Lille. 